\title{
Infectious respiratory disease: an update
}

\section{Jane E. Sykes}

Canine infectious respiratory disease complex (CIRDC) remains a major problem in shelter and boarding kennel environments, despite widespread vaccination against the disease. As a result of improvements in diagnostic testing, there is increasing awareness of mixed infections in affected animals. In environments such as shelters, co-infections with a variety of different viruses and bacteria may be more common that infections with a single pathogen. In addition, several pathogens have emerged in recent years as important contributors to CIRDC in kennel and shelter situations. Understanding the differential diagnosis for CIRDC is important because it aids selection of appropriate diagnostic tests, the design of rational therapy and permits institution of proper preventative measures for CIRDC. There are now over 10 organisms known to play a role in canine infectious respiratory disease. Bacterial causes of canine infectious respiratory disease include Bordetella bronchiseptica, Streptococcus equi subspecies zooepidemicus, and Mycoplasma spp. Viral causes of canine infectious respiratory disease include influenza viruses, canine distemper virus, canine respiratory coronavirus, canine parainfluenza virus, canine adenovirus (especially canine adenovirus-2), canine pneumovirus and canine herpesvirus.

Pathogens causing CIRDC can help each other to infect the host. Severe disease is more likely to be associated with co-infections. Single infections may be present in some animals that show no signs of illness. Prevention is assisted by proper attention to hygiene and quarantine, minimizing overcrowding within kennels and shelters, and use of vaccines for CIRDC. Because of growing concerns about antibiotic resistance, antibiotic treatment should be withheld unless dogs are systemically unwell and show signs of mucopurulent nasal discharge, lethargy or have evidence of secondary bacterial pneumonia. In this case, the possibility of distemper should also be considered. Dogs with uncomplicated 'kennel cough' (i.e. those that are otherwise bright and alert but just coughing) typically recover without treatment over 1-4 weeks. Cough suppression may be indicated to help the dog (and the owner) sleep at night. If antibiotics are deemed indicated, doxycycline should be considered as a first-line treatment because of its activity against Bordetella and mycoplasmas. The International Society for Companion Animal Infectious Diseases (ISCAID) recommends treatment for 7-10 days.

A diagnostic work-up is recommended for dogs with chronic upper respiratory signs, including imaging of the nasal cavity and rhinoscopy. Almost all dogs with chronic upper respiratory tract disease have disorders that are not effectively treated with antibacterials alone, such as nasal neoplasia, foreign bodies, lymphoplasmacytic rhinitis and sinonasal aspergillosis. Appropriate empirical therapy for dogs with pneumonia is either a beta-lactam (for dogs that are stable) or a combination of a beta-lactam or clindamycin and a fluoroquinolone. Shorter courses of antimicrobial therapy (10-14 days) should be considered when compared with those recommended previously (4-6 weeks), based on clinical reassessment. Dogs and cats with pyothorax should be treated with drainage and possibly surgical debridement, in combination with parenteral antimicrobial therapy that targets Gram-negative bacteria, Gram-positive bacteria, mycoplasmas and anaerobes pending the results of culture and susceptibility testing. The presence of anaerobes should always be assumed, even if they are not cultured.

\section{KEY LEARNING OBJECTIVES}

- Know the range of pathogens that can cause CIRDC and the importance of co-infections

- Know when to obtain a diagnosis and the optimum way to collect samples

- Understand current recommendations for treatment and prevention of infectious respiratory disease

\section{MULTIPLE CHOICE QUESTIONS}

1. Which of the following statements about CIRDC is FALSE?
(A) Bordetella bronchiseptica, canine distemper virus and canine respiratory coronavirus are examples of pathogens that can cause CIRDC
(B) Co-infections are common in CIRDC and contribute to disease severity
(C) None of the pathogens in the CIRDC can cause human disease
(D) Canine pneumovirus appears to be a
clinically significant CIRDC pathogen

2. Which of the following statements about treatment of CIRDC is FALSE?
(A) Antibiotic treatment for CIRDC should be withheld unless mucopurulent discharges and systemic signs of illness are present choice for empirical therapy of CIRDC is doxycycline
(B) When indicated, the antibiotic treatment of
(C) When indicated, the recommended duration of antibiotic treatment for CIRDC is $7-10$ days
(D) Amoxicillin is active against mycoplasmas and Bordetella

3. Which of the following statements about prevention of CIRDC is TRUE?
(A) Parenteral vaccines for Bordetella are more effective than mucosal vaccines
(B) Inadvertent administration of intranasal Bordetella vaccines via the subcutaneous route can cause death
(C) Intranasal Bordetella vaccines can cause human disease
(D) Quaternary ammonium compounds are suitable disinfectants for all pathogens in the CIRDC complex 